\title{
Lung Radiofrequency Ablation: Post-procedure Imaging Patterns and Late Follow-up
}

Jose Araujo-Filho ( $\square$ ariaraujocg@gmail.com )

Hospital Sirio-Libanes https://orcid.org/0000-0002-8627-3661

\section{Raonne Souza Almeida Alves Menezes}

Hospital Sirio-Libanes

\section{Natally Horvat}

Hospital Sirio-Libanes

\section{Pedro Sergio Brito Panizza}

Hospital Sirio-Libanes

João Paulo Giacomini Bernardes

Hospital Sirio-Libanes

Rodrigo Stanford Damasceno

Hospital Sirio-Libanes

Brunna Clemente Oliveira

Universidade de Sao Paulo Instituto de Radiologia

\section{Marcos Roberto Menezes}

Universidade de Sao Paulo Instituto do Cancer do Estado de Sao Paulo

\section{Research article}

Keywords: Lung Neoplasms, Ablation Techniques, Diagnostic Imaging, Multidetector Computed Tomography

Posted Date: August 17th, 2020

DOI: https://doi.org/10.21203/rs.2.22080/v2

License: (9) This work is licensed under a Creative Commons Attribution 4.0 International License. Read Full License

Version of Record: A version of this preprint was published at European Journal of Radiology Open on January 1st, 2020. See the published version at https://doi.org/10.1016/j.ejro.2020.100276. 
The authors have withdrawn this preprint from Research Square 